\title{
Labyrinthe
}

25 | 2006 (3)

La Bande dessinée : ce qu'elle dit, ce qu'elle montre

\section{Watchmen, à deux voix}

\section{Clotilde Thouret et Renaud Pasquier}

\section{(2) OpenEdition}

\section{Journals}

\section{Édition électronique}

URL : http://journals.openedition.org/labyrinthe/1414

DOI : $10.4000 /$ labyrinthe.1414

ISSN : 1950-6031

Éditeur

Hermann

\section{Édition imprimée}

Date de publication : 1 décembre 2006

Pagination : 69-82

ISBN : 2-9526131-2-5

\section{Référence électronique}

Clotilde Thouret et Renaud Pasquier, « Watchmen, à deux voix », Labyrinthe [En ligne], 25 | 2006 (3), mis en ligne le 28 mars 2010, consulté le 10 décembre 2020. URL : http://journals.openedition.org/

labyrinthe/1414; DOI : https://doi.org/10.4000/labyrinthe.1414

Ce document a été généré automatiquement le 10 décembre 2020.

Propriété intellectuelle 


\title{
Watchmen, à deux voix
}

\author{
Clotilde Thouret et Renaud Pasquier
}

1 A et B sont des lecteurs de Watchmen, d'Alan Moore et Dave Gibbons. Ils en parlent.

2 A - Que diriez-vous d'écrire un article sur les Watchmen pour Labyrinthe? Saisissons l'occasion : l'œuvre d'Alan Moore fête ses vingtans, comme le Dark Knight Returns de Frank Miller. Et comme le Dark Knight, Watchmen est une longue méditation sur les super-héros, maisaussi sur les comics, leur forme, leur histoire, à partir d'une base narrative très classique (l'élimination d'anciens héros costumés, qui cache un complot bien plus vaste). Il faudrait d'abord rappeler que le projet initial de Moore était de reprendre tels quels les personnages oubliés de la vieille "Charlton Line»; devant le refus des éditions $\mathrm{DC}^{1}$, propriétaire de Charlton (avec ce qu'il allait leur faire subir, ces super-héros seraient ensuite inutilisables pour de nouvelles aventures), il décidait d'inventer des personnages en faisant « muter » (c'est son mot) les héros Charlton ${ }^{2}$, et en les intégrant dans un monde réaliste. Le résultat obtenu était fascinant. Jamais un comics ne s'était construit aussi explicitement à partir d'une réflexion sur les comics et leur réception : Moore ne met pas en scène des personnages déjà existants, mais, dans le monde qu'il crée, certains individus deviennent des héros costumés parce qu'ils s'inspirent ${ }^{3}$ des super-héros de bandes dessinées qui ont exactement le même statut pour eux que pour nous lecteurs. C'est fascinant!

B - Bien sûr, les Watchmen, la réflexivité... Le sujet n'est-il pas un peu rebattu? Tant d'articles, d'universitaires ou de fans, en revues ou sur le Web, décortiquent les douze (ou six, en France) volumes pratiquement case par case, analysent la structure, les effets de symétrie, les sources, l'architecture subtile des narrations enchevêtrées, etc., etc. Dans quel but? Pour dire que c'est formidable, parce que c'est savant et compliqué. Discours de fan, cela. Si l'on s'occupe de Moore (et Dave Gibbons, ne l'oublions pas!), c'est pour s'interroger sur les enjeux politiques de Watchmen, pas pour en célébrer béatement la complexité. Quantàladimension " réflexive », ces références, ces traits rétro, ces couleurs criardes... Est-ce que c'est ça l'important ? N'y a-t-il pas là que clin d'œil érudit, du Tarantino en cases? Qu'en disent vraiment Moore et Gibbons, des comics? Je me demande... 
4 A - Oui, ces articles très descriptifs, très laudatifs (mais très instructifs, aussi)... Moore ne les attendait-il pas au tournant ? Écoutez-le :

"C'est du sur mesure pour un cours de fac, il y a tant de niveaux de lecture et de petits détails cachés en arrière-plan, tant d'allusions subtiles et de références érudites : les universitaires pourront travailler dessus pendant des années ${ }^{4} . »$

Pourtant, au-delà de cette facétieuse déclaration, je crois que la structure baroque qu'il a construite n'est pas qu'un piège à analystes compulsifs. Moore s'y complaît - disons même qu'il s'en grise -, c'est évident, mais elle construit aussi le sens. Ne brûlons pas les étapes. Que dit-il, que pense-t-il, me demandez-vous? D'abord il ne s'en tient pas à une sorte de « réflexivité » paresseuse et facile.

$\mathrm{B}$ - Cela reste à prouver...

7 A - J'y viens. Watchmen joue avec l'univers des comics sur des modes multiples et variés, et non pas seulement sur celui du « clin d'œil ». Il y a ainsi un puissant réseau d'échos et de parallélismes entre l'histoire (réelle) des comics de super-héros et l'histoire (fictionnelle) des justiciers masqués inventée par Moore. Reprenons brièvement les deux trames: la première équipe de héros (les Minutemen), dans laquelle se trouvaient notamment Blake (Le Comédien), Hollis Mason (le premier Hibou) et Sally Jupiter (la première Silk Spectre), a été rassemblée en 1938 c'est-à-dire à la date de la création de Superman - dans la réalité comme dans Watchmen, d'ailleurs. Après une période d'activité pendant la guerre, ils sont dispersés en 1949, soit, dans l'histoire des comics, à peu près à la fin de ce qu'il est convenu d'appeler le Golden Age ${ }^{5}$; la formation et les activités de la deuxième équipe (les Crimebusters) correspondent quant à elles au renouveau des comics de super-héros après la période de recul des années 1950. Enfin, l'époque où prend place le récit proprement dit (1985) est bien celui où renaît l'intérêt pour les super-héros - entre autres grâce à Watchmen; cela devient un peu vertigineux... Quant aux personnages, ils procèdent euxmêmes de relectures sans complaisance de super-héros classiques (plus connus en tout cas que leurs modèles officiels made in Charlton) : Blake, Le Comédien, barbouze fascisant, c'est évidemment une version grinçante du Captain America; Le Hibou, dépressif et ventripotent, c'est Batman; Jon, leDocteur Manhattan, seul surhomme stricto sensu, aux pouvoirs quasiment infinis, et qui perd peu à peu tout intérêt pour les simples mortels, c'est une variante de Superman le kryptonien, etc. Chaque figure enveloppe ainsi un discours critique (sur son modèle), bien plus riche et élaboré que bien de très sérieuses analyses.

B - Vous simplifiez. Ces personnages n'ont pas «un» modèle, ce sont plutôt des «précipités » de héros: Le Comédien, c'est autant Nick Fury que le Capitaine America, le Docteur Manhattan ressemble comme deux gouttes d'eau au Surfeur d'argent (éternelle victime de l'ingratitude viscérale des terriens...), etc. Le jeu pourrait continuer longtemps. Difficile alors d'établir le contenu critique de ces figures. Bref, vous ne me convainquez pas. Je me répète, mais Moore n'est-il pas qu'un fan au discours sophistiqué, mais creux ? Ne s'introduit-il pas lui-même dans le récit, sous la forme de ce gamin - bêtasse mise en abyme, en ce cas... - lecteur passionné d'une BD intitulée Tales of the Black Freighter? D'ailleurs cette série imaginaire, entre piraterie et fantastique horrifique, est encore un hommage cryptique à d'obscurs comics ${ }^{6}$ oubliés...

9 A - Précisément, la série du Black Freighter! Relisez le chapitre 5 du livre (fictionnel) Treasure Island Treasury of Comics (supposément publié à New York, un an avant les faits, donc en 1984), reproduit à la fin du chapitre V (justement) de Watchmen (au passage : insérer dans la Bande dessinée des articles critiques, interviews, extraits de livre, etc., 
c'est aussi une façon d'élargir et approfondir le discours). L'extrait est consacré à ce classique imaginaire des comics de pirates, les Tales of the Black Freighter. Ce texte intéressant, au ton sérieux, pas du tout un discours de fan, montre justement comment Moore évite les pièges de la vaine spécularité. Parce que cet article, c'est un journal de l'écriture des Watchmen. Suivez-moi bien. Premier indice: la série (Tales of the Black Freighter) est republiée en 1985, l'année oùjustement les justiciersmasqués refont surface... Ensuite: selon l'auteur, les scénarios de Max Shea s'améliorent et il prend en exemple le numéro 3. Or, que répond Moore aux questions sur la genèse de l'œuvre ${ }^{7}$ ? Que c'est en composant les premières planches du chapitre III qu'il a vraiment pris conscience des potentialités du scénario, et décidé de développer le comics de pirates lu par l'adolescent noir, de multiplier les échos, visuels et thématiques, d'une case à l'autre, d'une histoire à l'autre. De fait, Moore et Gibbons exploitent toutes les possibilités de la narration graphique, particulièrement celles offertes par le « caniveau ${ }^{8}$ » et la coexistence de l'image et du texte. Ils mêlent et entrecroisent les lignes narratives et les énonciations: le passé et le présent, les personnages secondaires et les personnage principaux, le monde de la fiction et l'imagination des personnages, le journal à l'énonciation mystérieuse et les voix de l'ici et maintenant, la tradition des comics et la nouveauté du «roman graphique $» . .$.

La densité du récit devient presque vertigineuse quand on trouve dans une même case $(\mathrm{XI}, 6)^{9}$ : une bulle qui correspond à l'histoire du naufragé, une image de la main du vendeur de journaux qui regrette de n'avoir pas quitté New York et un fragment d'image qui lance la séquence suivante, c'est-à-dire le récit autobiographique et épique de Veidt dans sa forteresse. Complexité ? Réflexivité ? Surtout un discours pratique sur l'art des comics - mais aussi sur leur histoire. Prenez par exemple les premières pages. La scène : Edward Blake est mort, deux enquêteurs cherchent des indices dans son appartement. En même temps que le point de vue monte du trottoir ensanglanté jusqu'à l'étage d'où a été lancée la victime, la voix de Rorschach, par le biais de son journal retranscrit, s'élève pour déplorer le vice des hommes et annoncer la fin du monde. Le texte n'est pas un commentaire de l'image, mais s'y articule: thématiquement, par le sang et la mort, et logiquement puisque Walter Kovacs (alias Rorschach) passe à ce moment même dans la rue en portant son affiche : "the End is nigh », « la fin est proche ». Ensuite, les images des flics dans l'appartement alternent (une case sur deux) avec les images en flashback de la scène du crime, oùl'on voit Blake se faire tabasser etbalancer à travers la baie vitrée sans jamais voir le visage ou le corps du meurtrier. Mais les réflexions des enquêteurs se poursuivent d'une case à l'autre, ouvrant ainsi sur une "chute» ironique: "rez-dechaussée, c'est parti ! », dit l'un des deux au moment de monter dans l'ascenseur, mais la phrase correspond à l'image qui montre Blake projeté dans le vide ${ }^{10}$.

11 À cela s'ajoute un effet de citation "graphique »: la séquence qui montre le crime, par son décor urbain et ses angles de vue exacerbés, reprend les techniques de dessin que recommande John Buscema pour dessiner Spiderman ${ }^{11}$ et qui sont des procédés récurrents et efficaces des comics de super-héros : ils donnent au lecteur un sentiment de vitesse et de vertige, et exaltent les exploits du héros. Mais Moore et Gibbons jouent ici sur une inversion, voire une perversion de la logique puisque ces codes sont utilisés pour montrer et souligner la chute du (super-)héros... Comme le dit le journaliste de l'article sur le Black Freighter, « leslecteurs qui abordaient la série en comptant y trouver de belles aventures de cape et d'épée étaient soit dégoûtés, soit fascinés par ce qui était souvent un 
commentaire pervers et noir sur la condition humaine » : remplacez «cape et d'épée » par « super-héros »...

B - Assez! Watchmen ce n'est pas ça! Pas que ça, du moins... Pas que du code perverti, pas un bidule postmoderne. D'abord, il n'est pas besoin de connaître par cœur l'histoire des comics pour goûter Watchmen. Ce que je lis, ce que je vois, moi, dans Watchmen, c'est undiscours politique. D'ailleurs, la politique, c'est important pour Moore, je vous le rappelle. Vous avez lu $V$ pour Vendetta? Vous vous êtes contenté du film...? V, c'est un super-héros anarchiste - il n'est d'ailleurs pas sans ressemblance avec le Docteur Manhattan, par son histoire et son statut, mais c'est son opposé sur un point essentiel : son rapport avec la société des humains qu'il tente de sauver, à sa manière. Ce sont des symétriques, des doubles inversés - mais reprenons le fil: un super-héros anarchiste, c'est une figure impossible ${ }^{12}$. Le super-héros se donne comme un garant non-légitime de l'ordre légitime et son personnage (plus encore quand c'est un personnage "réflexif » comme vous dites) pose nécessairement la question de l'ordre, social et politique.

Le problème des Watchmen, ce n'est pas les comics et leur histoire, c'est l'Ordre. Bienplus que le Pouvoir ${ }^{13}$. Qui a le droit de faire régner l'ordre? Quel ordre, d'ailleurs? Au nom de quoi? Who watches the Watchmen ${ }^{14}$ ? Et Moore a pris soin d'attacher ses principaux personnages à des ordres bien plus qu'à des (super)pouvoirs, et même de les définir, du moins quatre d'en- tre eux, par le choix d'un ordre singulier, dans un monde d'où s'est retirée toute Providence. Ainsi Rorschach veut-il faire régner l'ordre de la Morale, le Bien et le Mal éternels, impossibles à confondre. Le Comédien est l'agent de l'ordre arbitraire, celui de la force, de l'État; il est l'anti-Rorschach, même s'ils se retrouvent - alliance objective - dans la défense des valeurs traditionnelles et dans un certain désespoir cynique (Rorschach ou «le Mal finit toujours par l'emporter»; le Comédien ou «le monde est une farce ridicule »). Docteur Manhattan, évidemment, correspond à l'ordre de la nature ou plutôt il est l'ordre des atomes. Choisi par Adrian Veidt, l'ordre de la volonté et de l'intelligence, qu'il incarne, complète le tableau (ce dernier pourrait être, par son volontarisme, proche de Rorschach mais en devient l'opposé en se situant "par-delà le Bien et le Mal»). L'hésitation, ou plutôt le conflit entre ces quatre hypothèses, est la structure portante du récit.

14 A - Mais rien d'incompatible! Vous prolongez mon propos. Certes, il n'est pas indispensable de maîtriser toutes les références de Moore pour percevoir la force de l'œuvre, mais on ne peut nier que celles-ci nourrissent sa pensée. Accordez-moi que la réflexion politique de Moore et Gibbons en passe par une relecture de l'Histoire, et par l'invention d'un passé différent où les événements auraient suivi un tout autre cours, en somme que Watchmen est une « uchronie ».

B - Accordé.

6 A - Merci. L'œuvre est donc traversée par la question: comment écrire l'Histoire, ou mieux, penser l'Histoire à travers la Bande dessinée ? Certes pas en adoptant la méthode de l'illustration, celle de l'« Histoire en bandes dessinées », qui, petit, m'enthousiasmait en rendant visibles hautes figures et grands événements; mais bien en utilisant les moyens propres d'un art et les ressources d'une riche tradition, celle des comics. Moore construit son Histoire parallèle des États-Unis (donc du monde) sur des hypothèses sociopolitiques tirées des comics. Vous comprenez maintenant, la « réflexivité », le « comics qui parle de comics ", ce n'est pas un formalisme creux, c'est juste prendre au sérieux ces histoires en images - très au sérieux -, et donc tirer toutes les conséquences sociales et politiques de l'existence des super-héros que les fictions traditionnelles laissent, par 
convention, de côté - conséquences individuelles et collectives, je veux dire. Ici, par exemple, l'apparition du Docteur Manhattan, son contrôle absolu de l'atome, a modifié en profondeur non seulement sa vie sentimentale et affective, mais surtout l'évolution technologique, et, partant, la situation politique mondiale (Nixon deux fois réélu, les États-Unis vainqueurs au Viêt-Nam, etc.: tout cela non pas exposé lourdement, mais subtilement distillé dans la narration par allusions ou détails quasi-imperceptibles) et les sociétés elles-mêmes.

Moore met au jour les non-dits des vieux comics: forcément, il faut relire les grands auteurs, reconsidérer les personnages classiques et rendre visible leur part implicite, une certaine manière d'envisager la société qui allait de pair avec une vision de leur art. Par exemple, Rorschach, c'est Steve Ditko, le dessinateur de Spiderman, pas un gauchiste Ditko ${ }^{15}$, hein, loin de là, lisez ce qu'en disent Will Eisner et Frank Miller ${ }^{16}$, rien à voir avec Moore l'anarcho; et pourtant ce dernier a pour Ditko un profond respect, une vraie fascination, même ${ }^{17}$. Rorschach, c'est peut-être son plus beau personnage. À la fin... B - ... quand il enlève son masque, his face, comme il dit, un geste inconcevable, pas possible pour lui, mais si l'on regarde bien, les larmes dessinent encore les mêmes motifs, ceux du masque, sur son visage, his face...Quoi de plusémouvant?

19 A - Émouvant à pleurer... Je vais vous dire, il y a du Flaubert en Moore.

20 B - Vous dites?

21 A - Moore, c'est Flaubert. Il n'y a pas de sot sujet, «Yvetot vaut Constantinople », on peut faire une œuvre, une grande, avec un minable récit d'adultère provincial, avec des histoires de super-héros en collants flashy. C'est de l'anarchie aussi, anarchie esthétique, si vous voulez.

22 B - Oui, à ceci près que raconter les histoires de la Bovary, c'était pour Flaubert un défi intime, une gageure, il en avait des boutons, tandis que Moore en redemande, il adore ça les super-héros. N'empêche que, peut-être... écoutez ça :

«Le diable m'emporte si je ne me sens pas aussi sympathique pour les poux qui rongent un gueux que pour le gueux. Je suis sûr d'ailleurs que les hommes ne sont pas plus frères les uns des autres que les feuilles des bois ne sont pareilles : elles se tourmentent ensemble, voilà tout. Ne sommes-nous pas faits avec les émanations de l'univers ? [...] À force quelquefois de regarder un caillou, un animal, un tableau, je me suis senti y entrer. Les communications entr'humaines ne sont pas plus intenses. "

23 C'est une lettre de Flaubert à Louise Colet, du 26 mai 1853. Comparez :

« Tu as tenté de comparer la simple improbabilité de ton existence avec le chaos du monde que nous survolons... mais où sont les pinacles capables de rivaliser avec cet Olympe" (IX, 18). Ce sont les mots de Jon Osterman, le Docteur Manhattan, qui parle à Laurie Jupeczyk, sur Mars, en 1985. «J'ai marché sur la face du Soleil. J'ai assisté à des événements si infimes et si rapides que l'on peut à peine dire s'ils se sont produits. Mais toi... tu es un homme. Et l'homme le plus intelligent de ce monde ne signifie pas davantage pour moi que son plus intelligent termite » (XII, 18).

24 Ces phrases sont adressées à Ozymandias, Adrian Veidt, qui prétend tenir tête au même Docteur Manhattan. Même désintérêt pour la figure humaine, même fascination esthétique pour le réel dans ses plus infimes manifestations. Manhattan, Flaubert, même combat? Mais alors, peut-être que - à mon grand dam - la structure savante de Watchmen , le formalisme superlatif, c'est un peu la tentation Manhattan, représentée par l'horloge géante et inutile qu'il construit sur Mars, souvenir d'enfance, telle une Bande dessinée. 
Planète Mars Tour d'ivoire : aux autres, le souci de la communauté, la politique; mieux vaut construire, dans son coin, de merveilleuses machines, horloges, BD, ou « livres sur rien » qui tiennent " par la seule force interne du style ${ }^{18}$ ». C'est vrai, Watchmen exhibe les tendances esthétisantes de Moore l'anarchiste - dans $V$ pour Vendetta il est beaucoup plus assertif, voire propagandiste.

A - Et d'ailleurs la question de l'ordre et du chaos, chez Moore comme chez Flaubert, est esthétique autant que politique, par des moyens différents. Le romancier français fait sauter les liens logiques, façonne un récit désarticulé, paratactique, sans point de vue dominant; le scénariste anglais (avec son dessinateur) raffine la structure, summum de composition, d'ordre artistique, de maîtrise, au point de rejoindre le chaos..., et d'y perdre le lecteur.

27 A - Je m'explique. Tout indique l'ordre, dans Watchmen. D'abord la fiction même, intrigue linéaire où il s'agit bien de « rétablir l'ordre » en répondant aux questions : Qui veut tuer les justiciers masqués? Le monde finira-t-il ? La troisième guerre mondiale va-t-elle éclater? Ensuite dans la forme, avec la reprise du comics à l'ancienne avec ses couleurs vives et claires, son découpage rectangulaire et sans chichis - beau travail de Gibbons - et puis surtout l'accumulation de signes unifiants : citations lourdes de sens, constructions symétriques, jeux visuels ou thématiques qui structurent chaque chapitre et l'ensemble de l'œuvre (couvertures des chapitres, rimes visuelles transversales aux chapitres, échos entre l'histoire du naufragé et le récit global, etc., etc.). On tend forcément, dans la lecture, à synthétiser tout cela, à rassembler tous les indices, tous les signes, dans un même ensemble. Mais l'opération est contrecarrée par des forces centrifuges qui s'exercent sur le lecteur: d'abord, il est tiraillé entre la continuité narrative et l'autonomie picturale des planches...

B - Entre « linéaire » et « tabulaire ${ }^{19} \ldots$.., entre Moore et Gibbons ?

A - Tiens, oui, peut-être... tabulaire et linéaire, donc, mais pas seulement; à chacun des deux niveaux, le désordre s'installe, par la prolifération des signes, on l'a déjà dit, mais aussi celle des récits : l'intrigue linéaire, qui tend vers la fin, se ramifie en une série de narrations individuelles, de flashbacks, de points de vue (Rorschach, Manhattan, Veidt mais aussi Hollis Mason, Sally Jupiter, le psy) et des micro-récits (celui du couple de lesbiennes par exemple). Que se passe-t-il alors ? Le lecteur ne sait plus où donner de la tête, ses tentatives d'unification se heurtent à la multiplicité, son attention pour le récit principal est distrait par les intrigues secondaires, ses yeux s'arrêtent sur la structure générale d'une planche, puis errent à travers elle, isolent un strip, inspectent chaque case, mettent en relation des éléments à peine visibles; sur un plan interprétatif, il paraît impossible de poser des équivalences métaphoriques ou allégoriques pour stabiliser le sens, par exemple l'histoire du naufragé (dans le Black Freighter) fait tour à tour écho à celle de chacun des six personnages, les deux silhouettes d'Hiroshima peintes sur les murs de New York prennent chaque fois un sens différent, etc. L'ordre structurel, graphique et narratif, débouche donc, à force de raffinement, sur le désordre.

B - Disons plutôt que le travail de composition narrative et graphique conduit à sortir de l'opposition Ordre/Désordre, du moins à la neutraliser...

31 A - On peut le dire ainsi. Mais jamais les deux pôles ne disparaissent: on glisse toujours de l'un à l'autre, il est toujours question d'ordre et de désordre, dans la fiction, dans la 
structure, ça fluctue, ça bouge..., et cela malgré la précision quasi maladive, malgré les subtils jeux d'échos, toute cette élaboration cristalline que la fin devrait couronner.

B - «Devrait », oui... La fin, c'est un problème. Allez, disons-le sans ambages : la fin est nulle. Je ne comprends pas que même les fans les plus acharnés ne soient pas atterrés par le ridicule du « plan » machiavélique découvert par Rorschach et Le Hibou. Ce gros poulpe est aussi laid qu'inepte. Le produit du cerveau le plus puissant du monde? Lamentable, vraiment. Moore, n'a pas su finir, perdu dans cet entre-deux que vous évoquez. C'est triste.

33 A - Pas si vite! «Nulle», la fin? Pas celle de Moore. Celle d'Adrian Veidt, alias Ozymandias. L'homme le plus intelligent du monde? Le personnage le plus médiocre, plutôt. Le seul, d'ailleurs. B - Vous avez raison... Du coup, je reviens sur le problème de l'Ordre: Veidt le milliardaire, c'est surtout l'ordre capitaliste. Il a décliné son personnage en une infinité de produits dérivés, figurines, parfum, manuels de «bien-être», etc. ; l'Histoire en marche se traduit pour lui en termes de stratégies marketing $(\mathrm{X}, 8)$; il ne connaît d'autre réalité que celle projetée sur les écrans du monde entier, leur somme lui offrant, croit-il, une vision globale, parfaite. Il habite le cœur de la Société du spectacle, pourrait-on dire. D'ailleurs, on se demande si tous ses actes n'ont pas pour unique but d'accroître encore son immense fortune, et non de sauver l'humanité. Sa «Fin de l'Histoire », c'est celle de Francis Fukuyama ${ }^{20}$ - pas du tout la tasse de thé de Moore, à mon avis.

A - Pardon de revenir à ma marotte, mais relisons ce que dit Veidt au Hibou et à Rorschach: «Je ne suis pas le méchant d'un sérial de la Républic ${ }^{21}$ (XI, 27). Pas un méchant de «Bande dessinée », en somme : c'est justement pour cela qu'il est médiocre. La Bande dessinée, les comics, c'est autre chose, c'est plus sérieux, plus intelligent que lesfumeuses intrigues de Veidt. Veidt c'est de la BD, pas de la Bande dessinée. De tous, c'est le seul qui colle à son personnage, que sa double identité (c'est le seul à se démasquer publiquement, d'ailleurs) ne perturbe pas, il est sans tourments, sans mystères, aussi ridicule que son gros poulpe quand il se met solennellement en scène pour raconter sa vie (XI, 7)...

B - Un instant. Son problème n'est pas l'absence de souffrance en soi. Pourquoi est-il si médiocre ? Parce qu'il est seul, et n'en souffre pas. Rorschach est rongé jusqu'à la moelle par la solitude (il devient attendrissant dans sa maladroite déclaration d'amitié à Dan Dreiberg, le Hibou), la fatale solitude du Docteur Manhattan est réfutée par son amour jamais démenti pour Laurie, même Le Comédien n'est jamais seul, d'ailleurs il est père... Et puis, au-delà de Watchmen, le couple est une obsession d'Alan Moore. Parce que la société, donc la politique, cela commence avec deux individus. Relisez: le couple de lesbiennes, les disputes du psy et de sa femme, le naufragé du Black Freighter qui recherche son épouse. Et puis il y a Dan et Laurie...

A - C'est juste, le motif du «Deux» semble commander toute la structure ${ }^{22}$. Tout ce travail sur la symétrie, sur les doubles, peut-être aussi cette présence de l'homosexualité, qui intéresse beaucoup Moore (surtout féminine : il y a des personnages de lesbiennes dans V, dans From Hell, dans Top Ten et puis dans Lost Girls)..., le couple miroir, quirassemble deux éléments symétriques..., l'accord alors, l'harmonie ? Non. Le couple est toujours en crise, toujours entre fusion et rupture (V et Evey dans $V$ pour Vendetta, mais From Hell regorge aussi de ces couples tourmentés, tout comme Top Ten). Le couple 
homosexuel est un cas limite, de l'ordre de la perfection formelle, et en même temps il représente un scandale (surtout quand il est féminin), il est donc ferment de désordre.

B - Précisément, ne perdons pas le fil: la politique du couple, Dan et Laurie. La question politique commence avec le couple et les rapports de pouvoir qu'il induit (et c'est vrai que le couple de lesbiennes, c'est la contradiction en acte, Ordre/Désordre). Remarquable à cet égard que Laurie domine Dan..., et aussi que Dan et Laurie soient des héritiers, les personnages les plus marqués, voire entièrement définis par leur passé. Même si Laurie cherche à rompre avec celui-ci, elle finit par l'accepter, dans la souffrance, lorsqu'elle découvre l'identité de son père. Et Dan est un homme du passé, devenu héros masqué par admiration éperdue pour Hollis Mason, en bon lecteur de comics qu'il est; ce n'est pas un hasard s'il se parfume avec Nostalghia - un produit Veidt, tiens. C'est bien cela la nostalgie: vivre dans le passé, en sachant fort bien qu'il est mort à jamais. J'aime que l'histoire finisse avec eux (ou presque), comme un nouveau jeu contradictoire : l'Histoire n'est pas finie, l'ordre est toujours relancé par le désordre (en dernière instance, celui des papiers du News Frontierman oùla main candide du pigiste effleure la vérité, dans ladernière case), et le futur naîtra peut-être des héros du passé, Dan et Laurie. Surtout Laurie, d'ailleurs, surtout la femme.

A - Il en faut deux qui restent, c'est obligé. Pas de vie à un, pas de politique à un, pas d'art à un, pour que la fin ne soit qu'un début, il en faut deux, texte et image, Moore et Gibbons...

B - Ne vous emballez pas! Bon, on l'écrit cet article?

A - À deux voix?

B - À deux voix. On commence tout de suite.

\title{
BIBLIOGRAPHIE
}

\section{Bibliographie partielle d'Alan Moore}

\author{
Avec Dave Gibbons \\ Watchmen, 12 chapitres, New York, DC Comics, septembre 1986-octobre 1987. \\ Édition française en six volumes (les noms des personnages principaux tenant lieu de titre pour \\ chacun), trad. de Jean-Patrick Manchette, Paris, Zenda, 1987-1988 ; intégrale en deux volumes, \\ 1992 ; intégrale, Paris, Delcourt, 1998.
}

Autres œuvres citées ici (en édition française, quand elle existe)

Avec David Lloyd, V pour Vendetta, Paris, Zenda, 1989-1990 ; intégrale chez Delcourt, 1999.

Avec Eddie Campbell, From Hell, Paris, Delcourt, coll. « Contre-bande », 2000.

Avec Gene Ha et Zander Cannon, Top Ten, série publiée en 4 tomes, Paris, Semic, 2000-2004. 
Avec Melinda Gebbie, Lost Girls, Marietta, Top Shelf, 2006 (édition française annoncée pour 2008, chez Delcourt).

\section{NOTES}

1. DC Comics est l'un des deux géants de l'édition des comics, avec Marvel. Superman et Batman sont les figures de proue de DC, Spiderman, les Quatre Fantastiques et les X-Men, celles de Marvel.

2. "Toasting Absent Heroes. Alan Moore Discusses the Charlton-Watchmen Connection", entretien de Jon B. Cooke avec Alan Moore, Comic Book Artist, 9, août 2000, http:// www.twomorrows.com/comicbookartist/articles/09moore.html

3. À l'exception de Jon Osterman, le «Docteur Manhattan », ancien physicien nucléaire victime d'un accident lors d'une expérience dans son laboratoire, et devenu depuis une sorte de mutant doté de pouvoirs surhumains (c'est le seul parmi les héros de Watchmen).

4. «It's tailor-made for a university class, because there are so many levels and little background details and clever little connections and references in it that it's one that academics can pick over for years ", dans "The Alan Moore Interview ", entretien de Barry Kavanagh avec Alan Moore, 17 octobre 2000, section : "Watchmen, Microcosms and Details », Blather, http:// www.blather.net/arti-cles/amoore/watchmen3.html

5. Voir, entre autres, Les Daniels, Comix - A History of Comic Books in America, New York, Bonanza Books, 1971, Jean-Paul Jennequin, Histoire du Comic Book. I. Des origines à 1954, Paris, Vertige Graphic, 2002 et Jean-Paul Gabilliet, Des Comics et des hommes. Histoire culturelle des comic books aux États-Unis, Nantes,éd. du Temps, 2005. Sur les super-héros, voir Gérard Courtial, À la rencontre des super-héros, Marseille, Bédésup, 1984 ; Nicky Wright, The Classic Era of American Comics,Londres, Prion, 2000 ; ou Martin Winckler, Super-héros, Paris, Hachette, 2003.

6. En 1954, EC Comics, maison d'édition spécialisée dans les genres noirs, horreur ou sciencefiction qui sera ruinée par la campagne de Fredric Wertham (voir l'article «Du Voyou au Critique »), publie la série Piracy, qui semble être le modèle de Moore et Gibbons.

7. Voir les entretiens « Toasting Absent Heroes. Alan Moore Discusses the Charlton-Watchmen Connection », op. cit., et « Watchmen, Microcosms and Details», op. cit.

8. "Gutter» en anglais, soit l'espace qui sépare deux cases. Voir Scott McCloud, Understanding Comics, 1993, L'Art invisible. Comprendre la Bande dessinée, trad. Dominique Petitfaux, Chap. 3, ¿ Du sang dans le caniveau ", Vertige Graphic, 1999, p. 60-93.

9. On notera en chiffres romains le chapitre, suivant l'édition américaine en douze opuscules, puis en chiffres arabes le numéro de la planche (toujours visible, quelles que soient l'édition et la pagination, en bas à droite de chaque planche).

10. Au chapitre II (II, 27), on retrouve le même fonctionnement en échos, avec les mêmes images, mais cette fois, c'est le journal de Rorschach qui double le flashback et la tonalité est nettement plus pathétique.

11. Stan Lee et John Buscema, How to Draw Comics the Marvel Way, NewYork, Simon \& Schuster, 1978.

12. La série The Authority aborde le même paradoxe, en mettant en scène un groupe de superhéros à tendances anarchistes, voire révolutionnaires, qui ne cessent de s'interroger sur leur statut et leur fonction: l'influence de Watchmen et de $V$ pour Vendetta y est flagrante (de même que sur Stormwatch, «l'ancêtre » de The Authority). The Authority, créée par Warren Ellis et Bryan Hitch, trois séries publiées chez Soleil (2000-2001), puis chez Semic (2003-2004).

13. Comme le veut Thierry Groensteen, « De Watchmen à From Hell. Quelques obsessions et procédés récurrents dans l'œuvre d'Alan Moore ", $9^{e}$ Art. Les Cahiers du musée de la Bande dessinée, 6 , janvier 2001, p. 102-107. 
14. "Qui nous garde des Gardiens?", d'après la citation de Juvénal: "Quis custodiet ipsos custodes? " (Satire VI, «Sur les femmes", 347). Question lancinante dans l'œuvre, que l'on devine écrite sur les murs de New York (mais jamais entièrement visible).

15. Steve Ditko, co-créateur de Spiderman, a également travaillé pour Charlton, sur les personnages mêmes qui ont fourni à Moore la matière première de Watchmen. Il est aussi un lecteur notoire de la philosophe et romancière Ayn Rand, tenante d'un individualisme intégral accompli dans l'idéal héroïque, et dont le disciple le plus célèbre et le plus fervent aujourd'hui est Alan Greenspan, l'ancien gouverneur de la Federal Reserve, la Banque centrale américaine.

16. Voir Conversazione sul fumetto [Eisner/Miller], trad. ital. d'Andrea Plazzi, Bologne, Kappa Edizioni, 2005, p. 1112-114.

17. Voir « Toasting Absent Heroes. Alan Moore Discusses the Charlton-Watchmen Connection », op. cit.

18. Flaubert, lettre à Louise Colet du 16 janvier 1852 .

19. Voir Pierre Fresnault-Deruelle, «Du linéaire au tabulaire », Communications, 24, Paris, $1^{\mathrm{er}}$ trimestre 1976, p. 7-23.

20. The End of History and the Last Man, New York, Free Press, 1992, traduction française La Fin de l'Histoire et le dernier homme, Paris, Flammarion, 1992.

21. Les studios "Republic », créés en 1935, étaient célèbres pour leurs séries B, et notamment leurs adaptations de comics : la série « Captain Marvel » fut ainsi un grand succès.

22. Thierry Groensteen montre excellemment cette prégnance de la symétrie dans l'ensemble de l'œuvre, et son rôle d'interface entre propos politique et élaboration artistique, à partir d'une analyse du chapitre V («Fearful Symmetry» / «Terrible Symétrie »), dont le titre désigne la construction. «Cette insistance des motifs symétriques [...] peut [...] déboucher sur une interprétation symbolique globale de l'œuvre. La symétrie devient alors une catégorie abstraite, qui recouvre notamment le face-à-face de l'homme et de la femme, celui des superpuissances, et un jugement moral d'équivalence entre les criminels et les justiciers, lorsqu'ils usent de moyens comparables. La catégorie, en somme, qui relie et permet de penser ensemble la plupart des thèmes majeurs de Watchmen. "Système de la Bande dessinée, p. 117. Seulement il en fait un dispositif de lecture unifiant, alors que le travail de la symétrie (on préfèrera justement du " Deux ») dans Watchmen est aussi celui de la division qui distend les liens entre les composantes de la structure (et les éléments des sociétés humaines). En somme, Groensteen ne perçoit dans la symétrie qu'une force centripète, alors qu'elle est aussi centrifuge.

\section{AUTEURS}

\section{CLOTILDE THOURET}

Clotilde.thouret[at]wanadoo.fr

RENAUD PASQUIER

renaud.pasquier[at]gmail.com 